Tropical Journal of Pharmaceutical Research February 2019; 18 (2): 327-331

ISSN: $1596-5996$ (print); 1596-9827 (electronic)

(c) Pharmacotherapy Group, Faculty of Pharmacy, University of Benin, Benin City, 300001 Nigeria.

Available online at http://www.tjpr.org

Original Research Article

http://dx.doi.org/10.4314/tjpr.v18i2.15

\title{
Effect of Bushen Jiangu decoction on ovariectomy- induced osteoporosis in rats
}

\author{
Yi-fei Wang and Feng Xue* \\ Department of Orthopedics, Central Hospital of Shanghai Fengxian District, Shanghai 201499, China \\ ${ }^{*}$ For correspondence: Email: xuefeng5742@126.com; Tel: +86-21-57420713
}

Sent for review: 20 July 2018

Revised accepted: 9 January 2019

\begin{abstract}
Purpose: This study aimed to determine the therapeutic effect of Bushen Jiangu Decoction (BJD) on osteoporosis (OS) in rats.

Methods: The animals were divided into the following groups: control group, rats received no treatment; ovariectomy (OVX) group, rats subjected to OVX surgery and were treated with normal saline; OVX + Fosamax group, rats that received OVX and were treated with $2 \mathrm{mg} / \mathrm{kg} /$ week of Fosamax; low-dose BJD (40 mg/kg/day), medium-dose BJD (80 mg/kg/day) and high-dose BJD (160 mg/kg/day) groups, where rats received OVX surgery with BJD doses of 40,80 and $160 \mathrm{mg} / \mathrm{kg} /$ day, respectively. At four weeks after treatment with OVX, Fosamax or BJD was orally administered for four months. Bone mineral density (BMD) was assessed, while serum hormone, serum levels of follicle-stimulating hormone (FSH), alkaline phosphatase (ALP), estradiol (E2), luteinizing hormone (LH), osteocalcin (OC) and telopeptides of collagen type I (CTX) were measured by enzyme linked immunosorbent assay (ELISA).

Results: The results demonstrated that the reduced BMDs of $L 4$ femurs and vertebrae were inhibited by BJD. Furthermore, BJD significantly elevated the serum levels of FSH, E2 and LH in OS rats. Furthermore, the serum CTX, ALP and OC levels of these rats dramatically decreased, when compared to the OVX group.

Conclusion: These findings suggest that BJD can improve OVX-induced OS.
\end{abstract}

Keywords: Bushen Jiangu decoction, Osteoporosis, Bone mineral density, Serum biochemistry

\begin{abstract}
This is an Open Access article that uses a funding model which does not charge readers or their institutions for access and distributed under the terms of the Creative Commons Attribution License (http://creativecommons.org/licenses/by/4.0) and the Budapest Open Access Initiative (http://www.budapestopenaccessinitiative.org/read), which permit unrestricted use, distribution, and reproduction in any medium, provided the original work is properly credited.
\end{abstract}

Tropical Journal of Pharmaceutical Research is indexed by Science Citation Index (SciSearch), Scopus, International Pharmaceutical Abstract, Chemical Abstracts, Embase, Index Copernicus, EBSCO, African Index Medicus, JournalSeek, Journal Citation Reports/Science Edition, Directory of Open Access Journals (DOAJ), African Journal Online, Bioline International, Open-J-Gate and Pharmacy Abstracts

\section{INTRODUCTION}

The postmenopausal osteoporosis (OS), which is mainly characterized by degraded bone microstructure and reduced bone density, is considered as a systemic bone disease that mainly affects postmenopausal women $[1,2]$. Studies have shown that the levels of several proteins and serum markers may change during the development of postmenopausal OS, such as alkaline phosphatase (ALP), Vitamin D, estradiol (E2), C-terminal telopeptide of type I collagen (CTX) and interleukin-6 (IL-6).

The World Health Organization have reported that OS affects millions of people in the United States, Europe and Japan, and the incidence of OS dramatically increases along with age, which 
is dramatically higher in women over 50 years old $[3,4]$. Furthermore, this may bring huge physician and economic burden to patients and society [5-7]. Thus, searching for new therapeutic strategies for treating OS remains as a clinical challenge.

Recently, it was reported that hormone replacement therapy (HRT) has high exhibited efficacy and safety in the treatment of postmenopausal OS $[8,9]$. Furthermore, HRT has side effects in long-term treatment, such as injury to reproductive tissues [10-12]. Since traditional medicines for the treatment of OS have many disadvantages, such as side effects of gastrointestinal reactions and osteonecrosis of the jaw $[13,14]$, more new treatment methods are needed.

Bushen Jiangu decoction (BJD) is a traditional Chinese medicine that has been reported to be effective for treating some diseases, such as OS and sexual dysfunction [15]. However, few studies have focused on the underlying mechanisms of BJD in treating OS. The present study aimed to investigate the effectiveness of BJD on osteoporosis in a rat model, and it might provide deeper insights for the mechanism of BJD in treating OS and some new targets for OS.

\section{EXPERIMENTAL}

\section{Preparation of Bushen Jiangu decoction}

The materials of BJD comprised with Rehmannia glutinosa (Gaetn.) Libosch. ex Fisch. et Mey. (10 g), Epimedium brevicornu Maxim. (6 g), Cornus officinalis Sieb. et Zucc. $(10 \mathrm{~g})$, Common Yam Rhizome (8 g), and Angelica sinensis (4 g). These were obtained from the College of Pharmacy, Fudan University, Shanghai, China. The BJD was prepared according to the standard preparation method in the manufacturing laboratory of Shanghai Fengxian District Central Hospital.

\section{Animals}

Sixty female Sprague-Dawley (SD) rats (sixmonth old, 200-240 g) were purchased from the Shanghai Animal Center. These animals were given free access to food and water, according to guideline of Directive 2010/63/EU for handling animals [16] prior to commencement of the experiments. The present study was approved by the Animal Care and Use Committee of Shanghai Fengxian District Central Hospital (approval no. FDFX 2015-0014).
These rats were randomized into six groups ( $n=10$, each group): control group, rats received sham operation; OVX group, rats received OVX surgery and were treated with normal saline; OVX + Fosamax group, rats received OVX and were treated with $2 \mathrm{mg} / \mathrm{kg} /$ week of Fosamax; low BJD, middle BJD and high BJD groups, rats received OVX surgery with BJD doses of 40,80 and $160 \mathrm{mg} / \mathrm{kg} /$ day, respectively.

\section{Determination of bone mineral density (BMD)}

The BMD of the right femurs and L1-L5 vertebrae was determined through dual-energy X-ray absorptiometry (DEXA) scans (GE Healthcare, USA), according to manufacturer's instructions. All experiments were conducted in triplicate.

\section{Evaluation of serum FSH, LH and E2}

Serum E2, FSH and LH levels were detected by ELISA using commercial ELISA kits, according to manufacturer's instructions (all purchased from Jiancheng Biological Engineering Research Institute, Nanjing, China).

\section{Assessment of serum bone markers}

Serum ALP, OC and CTX-I levels were measured using commercial ELISA kits, according to manufacturer's instructions (all purchased from Jiancheng Biological Engineering Research Institute, Nanjing, China).

\section{Statistical analysis}

Continuous data are expressed as mean \pm standard deviation (SD). Comparisons were analyzed using one-way ANOVA, followed by Tukey's post-hoc test. A $P$-value $<0.05$ was considered as significant. All calculations were performed using SPSS 18.0.

\section{RESULTS}

\section{BJD enhanced the BMD of L1-L5 vertebrae and femurs in OS rats}

As shown in Table 1, the BMD values of rats in the OVX group significantly decreased, when compared to the control group $(p<0.05)$. Furthermore, BJD treatment dramatically increased BMD, and this was decreased by OVX $(p<0.05)$. The effect was dose-dependent. Similarly, Fosamax also obviously elevated BMD $(p<0.05)$, and the effect was similar to the high concentration of BJD. These results suggested that BJD could enhance the reduced BMD in OS rats. 
Table 1: BMD of rats in the different groups $(n=10)$

\begin{tabular}{|c|c|c|}
\hline Group & $\begin{array}{l}\text { BMD of the vertebra } \\
\left(\mathrm{g} / \mathrm{cm}^{2}\right)\end{array}$ & $\begin{array}{c}\text { BMD of the femur } \\
\left(\mathrm{g} / \mathrm{cm}^{2}\right)\end{array}$ \\
\hline Control & $0.89 \pm 0.05^{\circ}$ & $0.53 \pm 0.03$ \\
\hline OVX & $0.21 \pm 0.04$ & $0.17 \pm 0.04$ \\
\hline Fosamax & $0.36 \pm 0.04^{*}$ & $0.27 \pm 0.04^{\star}$ \\
\hline Low BJD & $0.26 \pm 0.03$ & $0.16 \pm 0.03$ \\
\hline Middle BJD & $0.34 \pm 0.04^{*}$ & $0.25 \pm 0.03^{*}$ \\
\hline High BJD & $0.68 \pm 0.04^{\pi}$ & $0.47 \pm 0.03^{\pi}$ \\
\hline
\end{tabular}

\section{BJD elevated FSH, LH and E2 in OS rats}

In order to further investigate the effect of BJD on $\mathrm{OS}, \mathrm{FSH}, \mathrm{LH}$ and E2 in the different groups of rats, these were measured by ELISA. The results revealed that all indexes were significantly reduced by OVX $(P<0.01)$. However, the treatment with all concentrations of BJD, as well as the treatment with Fosamax, significantly increased hormone levels, but were decreased by OVX $(P<0.05)$, and this effect occurred in a dose-dependent manner (Table 2). This indicates that BJD can elevate the hormone levels of OS rats.

\section{BJD decreased ALP, CTX-I and OC in OS rats}

Lastly, alteration of ALP, CTX-I and OC in rats in the different groups were determined. As shown in Table 3, significantly higher serum levels of ALP, CTX-I and OC were observed in rats in the OVX group $(P<0.05)$. However, all concentrations of BJD, as well as Fosamax, dramatically decreased the levels of the above biomarkers, but were increased by OVX $(P<0.01)$. These results suggest that BJD can also decrease the levels of ALP, CTX-I and OC in OS rats.

Table 2: Serum hormone levels of rats in the different groups $(n=10)$

\begin{tabular}{lccc}
\hline Group & E2 $(\mathbf{p m o l} / \mathbf{L})$ & FSH $(\mathbf{I U} / \mathbf{L})$ & LH $(\mathbf{m l U} / \mathbf{m L})$ \\
\hline Control & $6.85 \pm 0.22^{\prime \prime}$ & $2.56 \pm 0.07^{\prime \prime}$ & $2.87 \pm 0.13^{\circ}$ \\
OVX & $1.43 \pm 0.15$ & $0.57 \pm 0.04$ & $1.23 \pm 0.08$ \\
Fosamax & $4.15 \pm 0.26^{\circ}$ & $1.53 \pm 0.06^{\circ}$ & $2.53 \pm 0.05^{\circ}$ \\
Low BJD & $2.38 \pm 0.28$ & $0.83 \pm 0.05$ & $1.73 \pm 0.10$ \\
Middle BJD & $3.34 \pm 0.26^{\circ}$ & $1.37 \pm 0.04^{\circ}$ & $2.52 \pm 0.08^{\circ}$ \\
High BJD & $4.78 \pm 0.16^{* *}$ & $1.72 \pm 0.04^{* *}$ & $2.64 \pm 0.06^{*}$
\end{tabular}

$P<0.05$ and " $p<0.01$ vs. the OVX group

Table 3: Serum ALP, CTX-I and OC levels of rats in the different groups $(n=10)$

\begin{tabular}{|c|c|c|c|}
\hline Group & ALP (U/L) & CTX-I (ng/mL) & $\mathrm{OC}(\mathrm{ng} / \mathrm{mL})$ \\
\hline Control & $113.4 \pm 3.9$ & $38.7 \pm 1.3$ & $57.3 \pm 2.5$ \\
\hline OVX & $242.3 \pm 6.1^{* \pi}$ & $75.3 \pm 1.4^{\pi x}$ & $83.6 \pm 2.2^{\pi *}$ \\
\hline Fosamax & $173.6 \pm 6.3^{\prime \prime}$ & $50.4 \pm 2.1^{\prime \prime}$ & $56.5 \pm 1.7^{\prime \prime}$ \\
\hline Low BJD & $234.1 \pm 5.4$ & $70.7 \pm 2.2$ & $76.6 \pm 2.1$ \\
\hline Middle BJD & $186.6 \pm 4.4$ & $65.3 \pm 1.6^{x}$ & $73.5 \pm 1.6$ \\
\hline High BJD & $1365 \pm 4.3^{\prime \prime}$ & $46.8 \pm 1.8^{\prime \prime}$ & $63.7 \pm 1.9^{\prime \prime}$ \\
\hline
\end{tabular}




\section{DISCUSSION}

Despite numerous studies on postmenopausal OS, better understandings and new treatment approaches for this disease are still needed [16]. In recent decades, more and more studies have demonstrated the effectiveness of Chinese medicinal herbal extracted in treating bone protection, including OS [17-22]. However, to date, few studies have focused on the effects and underline mechanism of BJD in treating OS. In the present study, it was demonstrated that BJD could enhance the OVX-induced BMD reduction, and decrease the OVX-induced ALP, CTX-I and OC levels in OS rats.

A decreased BMD is one of the main results in postmenopausal OS women, and is also a main risk factor in OS caused by fractures [23]. In the present study, it was revealed that BMD significantly reduced in OVX-treated rats, which was in consistent with previous studies [22,24]. Meanwhile, treatment of BJD significantly enhanced BMD.

It is widely accepted that a reduction in $\mathrm{E} 2 \mathrm{FSH}$ and $\mathrm{LH}$ is a signal of OS [25]. In the present study, decreased E2, FSH and $\mathrm{LH}$ were also observed in OS rats and BJD could reverse the effects by OVX, which was consistent with other researches. In addition, OVX treatment induced an increasing ALP, CTX-I and OC expression, which were biochemical bone turnover markers. It has been considered in OS patients that ALP, CTX-I and OC levels are all elevated [26]. In the present study, $160 \mathrm{mg} / \mathrm{kg}$ of BJD significantly decreased serum ALP, CTX-I and OC levels, indicating that BJD might improve OS by elevating the levels of bone turnover markers.

\section{CONCLUSION}

The results of this study reveal that BJD enhance OVX-induced BMD reduction, and decreases OVX-induced ALP, CTX-I and OC levels in OS rats. The findings also provide a deeper insight into the mechanism of BJD in OS, as well as new research targets for OS.

\section{DECLARATIONS}

\section{Conflict of Interest}

No conflict of interest associated with this work.

\section{Contribution of Authors}

We declare that this work was done by the authors named in this article and all liabilities pertaining to claims relating to the content of this article will be borne by the authors. Feng Xue conceived and designed the study, Yi-fei Wang collected and analyzed the data. All two authors wrote and approved the manuscript for publication.

\section{REFERENCES}

1. NIH Consensus Development Panel on Osteoporosis Prevention, Diagnosis, and therapy. Osteoporosis prevention, diagnosis, and therapy. $J$ Am Med Ass 2001; 285: 785-795.

2. Eastell R, Black DM, Boonen S. Effect of once-yearly zoledronic acid five milligrams on fracture risk and change in femoral neck bone mineral density. J Clin Endo Metabol 2009; 94: 3215-3225.

3. Burge $R$, Dawson-Hughes $B$, Solomon $D H$. Incidence and economic burden of osteoporosis-related fractures in the United States 2005-2025. J Bone Min Res 2007; 22: 465-475.

4. Omsland TK, Emaus N, Tell GS. Mortality following the first hip fracture in Norwegian women and men. A NOREPOS study. Bone 2014; 63: 81-86.

5. Marcus R. An expanded overview of postmenopausal osteoporosis. J Mus Neuronal Int 2002; 2: 195-197.

6. Sugerman DT. Osteoporosis JAMA 2014; 311: 104-105.

7. Johnell $O$, Kanis JA. An estimate of the worldwide prevalence and disability associated with osteoporotic fractures. Osteoporosis Inter 2006; 17: 1726-1733.

8. Stevenson JC. Justification for the use of HRT in the long-term prevention of osteoporosis. Maturitas 2005; 51: 113-126.

9. Prelevic GM, Kocjan T, Markou A. Hormone replacement therapy in postmenopausal women. Minerva Endocrinologica 2015; 30: 27-36.

10. Gray S. Breast cancer and hormone-replacement therapy: the Million Women Study. Lancet 2013; 362: 1332-1333.

11. Orija IB, Mehta A. Hormone replacement therapy: current controversies. Clinical Endocrinol 2013; 59: 657-658.

12. Lacey JV, Mink PJ, Lubin JH. Menopausal hormone replacement therapy and risk of ovarian cancer. J Ame Med Asso 2012; 288: 334-341.

13. Rossouw JE, Anderson GL, Prentice RL. Risks and benefits of estrogen plus progestin in healthy postmenopausal women: principal results from the Women's Health Initiative randomized controlled trial. $J$ Ame Med Assoc 2012; 288: 321-333.

14. Lee JK, Kim KW, Choi JY. Bisphosphonates-related osteonecrosis of the jaw in Korea: a preliminary report. $J$ Korean Asso of Oral and Maxi Surgeons 2013; 39: 9-13.

15. Donnapee S, Li J, Yang X. Cuscuta chinensis Lam.: A systematic review on ethnopharmacology, phytochemistry and pharmacology of an important traditional herbal medicine. J Ethnopharmacol 2014; 18: 292-308. 
16. European Commission [homepage on the internet]. Directive 2010/63/EU on the protection of animals used for scientific purposes [cited 2013 Jan 16]. Available from:http://ec.europa.eu/environment/chemicals/lab_ani mals/legislation_en.htm.

17. Lim D W, Kim Y T. Anti-osteoporotic effects of Angelica sinensis (Oliv.) Diels extract on ovariectomized rats and its oral toxicity in rats. Nutrients 2014, 6(10): 4362.

18. Lim $D W$, Kim J G, Lee $Y$, et al. Preventive effects of Eleutherococcus senticosus bark extract in OVX induced osteoporosis in rats. Molecules, 2013, 18(7):7998-8008.

19. Zhang L, Yue $X X$, Zhang $L$, et al. Anti-osteoporosis effect of Cistanche deserticola Ma extract in ovariectomized rats. Trop J Pharm Res 2016, 15(9): 1929-1933.

20. Wu $S H$, Zheng $C P$, Chen $S Y$, et al. Anti-thrombotic effect of Carthamus tinctorius Linn extracts in rats. Trop J Pharm Res 2014, 13(10):1637-1640.
21. Cai $Y$, Zhang $X$. Protective effect of Rhizoma drynariae extract on osteoporosis in ovariectomized rat model. Trop J Pharm Res 2016; 15(7): 1447-1452.

22. Wronski TJ, Dann LM, Scott KS. Endocrine and pharmacological suppressors of bone turnover protect against osteopenia in ovariectomized rats. Endoc 2009; 125: 810-816.

23. Turner RT, Vandersteenhoven JJ, Bell NH. The effects of ovariectomy and 17 beta-estradiol on cortical bone histomorphometry in growing rats. J Bone Mini. Res 2010; 2: 115-122.

24. Dai $S Q$, Yu LP, Shi $X$, Wu $H$, Shao $P$, Yin GY. Serotonin regulates osteoblast proliferation and function in vitro. Braz J Med Biol Res 2014; 47: 759-765.

25. Dallas SL, Prideaux M, Bonewald LF. The osteocyte: an endocrine cell and more. Endocr Rev 2013; 34: 658690.

26. Carson JA, Baltgalvis $K A$. Interleukin 6 as a key regulator of muscle mass during cachexia. Exerc Sport Sci Rev 2010; 38: 168-176. 